\title{
First principles calculations on structure, bonding, and vibrational frequencies of $\mathrm{SiP}_{2}$
}

\author{
F. Bachhuber, ${ }^{1}$ J. Rothballer, ${ }^{2}$ F. Pielnhofer, ${ }^{2}$ and R. Weihrich ${ }^{1,2, a)}$ \\ ${ }^{1}$ Universität Regensburg, Institute of Inorganic Chemistry, Universitätsstr. 31, 93040 Regensburg, Germany \\ ${ }^{2}$ Universität Ulm, Institute of Inorganic Chemistry - Materials and Catalysis, Albert-Einstein-Allee 11, \\ 89081 Ulm, Germany
}

(Received 5 April 2011; accepted 8 August 2011; published online 28 September 2011)

\begin{abstract}
Pyrite type $\mathrm{SiP}_{2}$ is reinvestigated by first principles calculations on various levels of functionals including local density approximation, generalized gradient approximation, Becke-Lee-Yang-Parr hybrid functional, and the Hartree-Fock method. $\mathrm{SiP}_{2}$ is seen as a model compound with molecular $[\mathrm{P}-\mathrm{P}]$ entities and $\left[\mathrm{SiP}_{6}\right]$ octahedra. Structure and bonding are addressed by electronic structure calculations. Special attention is spent on $\mathrm{P}-\mathrm{P}$ and $\mathrm{Si}-\mathrm{P}$ bonds in terms of bond lengths and respective stretching modes from simulated Raman spectra. The electronic structure is analyzed in both direct and momentum space by the electron localization function and site projected density of states. The main goals of this work are to understand the nature of chemical bonding in $\mathrm{SiP}_{2}$ and to compare and contrast the different methods of calculation. (c) 2011 American Institute of Physics. [doi:10.1063/1.3633951]
\end{abstract}

\section{INTRODUCTION}

There is a big variety of different phosphorus compounds and even new modifications of the element are still being discovered. ${ }^{1,2}$ Continuing interest in chemistry and bonding of phosphorus particularly exists for the solid state. Most phosphorus structures tend to be connected by single bonds both in its modifications like in $\mathrm{P}_{4}$ or recently found phosphorus nanorods. ${ }^{3}$ Charged partial structures of $\mathrm{P}$ are well known for various phosphide compounds such as $\mathrm{Cu}_{2} \mathrm{P}_{20}$ (Ref. 4) or $\mathrm{Cu}_{2} \mathrm{I}_{2} \mathrm{P}_{14}, 5,6$ the first binary polyphosphide with entirely preserved channels of phosphorus chains. The orthorhombic modification of $\mathrm{SiP}_{2}$ forms related $\mathrm{Si}-\mathrm{P}$-channels. It was discovered by Wadsten ${ }^{7}$ in 1967 by temperature gradient solid state synthesis.

Here, we focus on the cubic modification of $\mathrm{SiP}_{2}$ that is probably the simplest polyphosphide with $\mathrm{P}_{2}$ dumbbells in a pyrite type structure. It serves as a prototype for novel isoelectonic dipnictices like $\mathrm{SiN}_{2}$ that was predicted as a ultrahard large gap semiconductor. ${ }^{8} \mathrm{SiP}_{2}$ was first synthesized by Tanaka9 ${ }^{9}$ from high temperature and high pressure solid state synthesis, later by halogen transport from the elements under autogenous pressure. ${ }^{10}$ The key question to understand is the chemical bonding of $\mathrm{SiP}_{2}$ which seems to be straightforward in the first place but presents some surprises. The pyrite type structure with 6-fold coordination of Si favors a Zintl like description. ${ }^{11}$ Electron transfer from less electronegative $\mathrm{Si}$ atoms to more electronegative $\mathrm{P}$ atoms results in $\mathrm{Si}^{4+}$ and $\left[\mathrm{P}_{2}\right]^{4-}$ ions (isoelectronic to $\left[\mathrm{S}_{2}\right]^{2-}$ in $\mathrm{FeS}_{2}$ ). However, light elements such as $\mathrm{Si}$ and $\mathrm{P}$ are expected to form at least partially covalent bonds as concluded by von Schnering from $\mathrm{X}$-ray diffraction data. ${ }^{12}$

\footnotetext{
a) Author to whom correspondence should be addressed. Electronic mail: richard.weihrich@chemie.uni-r.de.
}

Looking at compounds that are isostructural to pyritetype $\mathrm{SiP}_{2}$, such as $\mathrm{NiP}_{2}, \mathrm{PtP}_{2}$, or pyrite itself $\left(\mathrm{FeS}_{2}\right)$, it is remarkable that these compounds are semiconductors. Nevertheless, $\mathrm{SiP}_{2}$ was characterized as a semimetal with a nearly filled Brillouin zone. ${ }^{10}$ The possibility of a semiconducting status was ruled out due to a rising electrical resistivity with increasing temperature. Furthermore, galvanomagnetic and optical measurements predicted the presence of both holes and electrons. From the analysis of Fermi surfaces, Farberovich ${ }^{13}$ proposed metallic type conductivity for $\mathrm{SiP}_{2}$. When it comes to electronic structure calculations, just little attention has been paid to $\mathrm{SiP}_{2}$ to date. Indeed, early band structure calculations from 1974 and 1976 (Refs. 14 and 15) characterized $\mathrm{SiP}_{2}$ as semimetal. However, the true nature of chemical bonding in $\mathrm{SiP}_{2}$ and the reason for the metallic behavior is still unclear.

The bonding situation was reconsidered by our group lately ${ }^{16}$ in terms of calculations of IR/Raman frequencies with modern $a b$ initio methods that allow for an enhanced treatment of exchange and correlation effects. As it can be seen by a more extensive approach in this work, these effects turn out to be crucial for the bond description in $\mathrm{SiP}_{2}$. It was carried out systematically due to calculated bond lengths, electronic structures in direct (electron localization function, ELF) and reciprocal space, and vibrational frequencies. From subsequently presented results, one can gain a deeper insight into the bonding situation in $\mathrm{SiP}_{2}$. Furthermore, different basis sets and methods of calculation were evaluated in terms of correctness and accuracy.

\section{METHODS}

Calculations have been performed with the CRYSTAL98 (Ref. 17) and the CRYSTAL06 codes. ${ }^{18}$ With the aid of these codes, the electronic structure was computed 
according to a linear combination of Gaussian type functions scheme. Full geometry optimizations, simulation of vibrational spectra, and electronic band structure calculations were done within the local density approximation (LDA), ${ }^{19}$ the Perdew-Burke-Ernzerhof generalized gradient approximation (GGA-PBE), ${ }^{20}$ and the Becke-Lee-Yang-Parr hybrid functional (B3LYP) (Ref. 21) of the Kohn-Sham density functional theory (DFT). ${ }^{22}$ By comparison, periodic Hartree-Fock (HF) (Ref. 23) calculations were performed as this method uses exact exchange but neglects correlation. The ELF and data related to the theory of atoms in molecules and crystals (AIM) (Ref. 24) were computed from CRYSTAL 98 density with TOPOND98. ${ }^{25}$

Pack-Monkhorst and Gilat nets were calculated with shrinking factors of (6 12) (119 k-points). The convergence criterion with respect to the total energy was set to $10^{-8}$ a.u. Anderson's method ${ }^{26}$ of mixing the Fock and Kohn-Sham matrix was used. All electron basis sets were applied with reoptimized outer shells. ${ }^{16,27}$ To examine the impact of the basis sets on the calculated geometrical parameters, calculations of $\mathrm{P}-\mathrm{P}$ and $\mathrm{Si}-\mathrm{P}$ distances were exemplarily performed with different basis sets (Fig. 2). For P, $88-311 \mathrm{G}^{*}$ was used. For Si, BS1 (88-31G*) includes less diffuse shells whereas there are additional shells implemented in BS2 (88-311G*) leading to a more diffuse character. For BS3 (88-1111G*), the basis set for each method was optimized separately. From spin polarized calculations, no magnetic ground state was detected.

\section{Results I: Crystal structure optimisations}

From a local point of view, the bond analysis refers to $\mathrm{P}-$ $\mathrm{P}$ dumbbell units and $\mathrm{SiP}_{6}$ octahedra as the 2 important building blocks of pyrite $\left(\mathrm{FeS}_{2}\right)$ type $\mathrm{SiP}_{2}$ (space group $\mathrm{Pa} \overline{3}$ ). Si atoms form an $f c c$ partial structure (crystallographic position 4a) like $\mathrm{Na}$ in $\mathrm{NaCl}$. Instead of single-atom anions, phosphorus dumbbells are located in the $\mathrm{Si}_{6}$ octahedral vacancies. This results in 4 formula units (FU) $\mathrm{SiP}_{2}$ per unit cell. With the $\mathrm{P}$ atoms on sites 8c (x, x, x) (see also Ref. 28), a coordination of $\mathrm{P}$ atoms by $3 \mathrm{Si}$ and $1 \mathrm{P}$ neighbor is realized that reminds of carbon in an ethane molecule (Fig. 1, left). From the point of view of $\mathrm{Si}$ atoms, a $3 \mathrm{D}$ network of $\mathrm{Si}\left[\mathrm{P}_{2}\right]_{6}$ polyhedra is formed

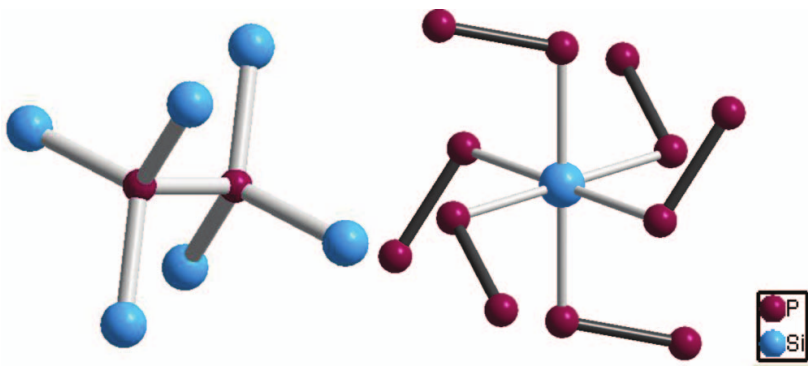

FIG. 1. Structural fragments in $\mathrm{SiP}_{2}$. (Left) Coordination sphere of a phosphorus dumbbell. (Right) Extended coordination sphere of a $\mathrm{Si}$ atom (the lines between the atoms are no bonds but serve as guide for the eye).

(Fig. 1, right). Contrary to edge-linked octahedra in the rock salt structure, the phosphorus dimers in $\mathrm{SiP}_{2}$ cause a deviation of $\mathrm{P}-\mathrm{Si}-\mathrm{P}$ angles from $90^{\circ}$ to $94^{\circ}$ and corner-linking of the resulting trigonal antiprism. Questions on chemical bonding refer to the covalence of the $\mathrm{Si}-\mathrm{P}$ bonds and whether the $\mathrm{P}_{2}$ dumbell can be seen as $\left[\mathrm{P}_{2}\right]^{4-}$ with the 3 lone pairs pointing towards $\mathrm{Si}^{4+}$ ions.

As a first approach to evaluate the bond types in $\mathrm{SiP}_{2}$, atomic distances are analyzed (Table I, Fig. 2). Calculated bond lengths $\mathrm{P}-\mathrm{P}$ and $\mathrm{Si}-\mathrm{P}$ are in good agreement with experimental data on all applied levels of theory and basis sets. The overall deviation of the geometrical parameters ranges between $-0.91 \%$ (for the LDA calculated $\mathrm{Si}-\mathrm{P}$ distance) and $1.71 \%$ (for the B3LYP calculated lattice constant). The maximum deviation between results obtained with different basis sets is about one percent. Consequently, the effect of the basis set is supposed to be relatively small with respect to the accuracy of the calculations. All following results are presented on the basis of BS2 only as BS2 calculations did not cause any problems with the convergence of energy with either method. As a reference, highly accurate experimental data from neutron and x-ray diffraction at $60 \mathrm{~K}$ (Ref. 29) are used. Thus, the $\mathrm{P}-\mathrm{P}$ length in $\mathrm{SiP}_{2}$ of $2.17 \AA$ is in the range of short $\mathrm{P}-\mathrm{P}$ bonds known for white $\mathrm{P}_{4}$ (Ref. 30) or $\mathrm{Cu}$ phosphides ${ }^{4,5}$ but shorter than in black $\mathrm{P}(2.22 \AA),{ }^{31}$ or in recently discovered fibrous $\mathrm{P}(>2.23 \AA) .{ }^{1}$ The $\mathrm{Si}-\mathrm{P}$ bonds $(2.39 \AA)$, on the other hand, are significantly longer than the sum of the covalent radii $(2.28 \AA)$ of $\mathrm{P}(1.11 \AA)$ and $\mathrm{Si}(1.17 \AA)$.

TABLE I. Calculated crystal structure data

\begin{tabular}{|c|c|c|c|c|c|c|c|c|}
\hline $\mathrm{SiP}_{2}$ & $\mathrm{a} / \AA ̊$ & $\mathrm{X}(\mathrm{P})$ & $d(\mathrm{Si}-\mathrm{P}) / \AA$ & $d(\mathrm{P}-\mathrm{P}) / \AA$ & $\mathrm{V} / \AA^{3}$ & $d(\mathrm{Si}-\mathrm{P}) / d(\mathrm{P}-\mathrm{P})$ & $\begin{array}{l}<\mathrm{P}-\mathrm{Si}-\mathrm{P} /{ }^{\circ} \\
<\mathrm{Si}-\mathrm{P}-\mathrm{P} /{ }^{\circ}\end{array}$ & $\Delta \mathrm{E}_{\mathrm{gap}} / \mathrm{eV}$ \\
\hline Expt. $(298 \mathrm{~K})^{29}$ & 5.707 & 0.391 & 2.397 & 2.162 & 185.88 & 1.109 & $\begin{array}{r}93.9 \\
103.6\end{array}$ & $\ldots$ \\
\hline Expt. $(60 \mathrm{~K})^{29}$ & 5.681 & 0.391 & 2.386 & 2.157 & 183.35 & 1.106 & $\begin{array}{r}93.9 \\
103.6\end{array}$ & $\ldots$ \\
\hline $\mathrm{HF}$ & 5.739 & 0.392 & 2.413 & 2.156 & 189.01 & 1.119 & $\begin{array}{r}93.8 \\
104.0\end{array}$ & 4.8 \\
\hline LDA & 5.656 & 0.390 & 2.375 & 2.155 & 180.96 & 1.102 & $\begin{array}{r}93.9 \\
103.5\end{array}$ & $\ldots$ \\
\hline PBE/GGA & 5.737 & 0.391 & 2.410 & 2.174 & 188.82 & 1.109 & $\begin{array}{r}93.9 \\
103.6\end{array}$ & $\ldots$ \\
\hline B3LYP & 5.778 & 0.391 & 2.430 & 2.173 & 192.95 & 1.118 & $\begin{array}{r}93.9 \\
103.6\end{array}$ & $\ldots$ \\
\hline
\end{tabular}




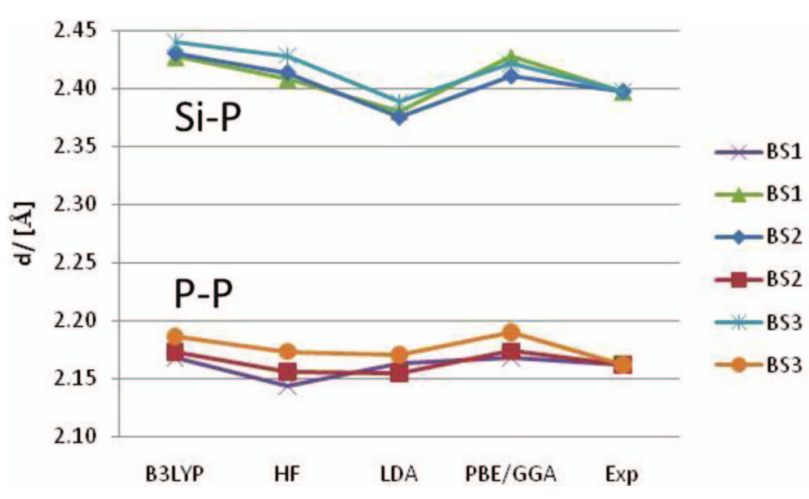

FIG. 2. The Si-P (upper) and P-P (lower) distances are displayed against different methods of calculation (B3LYP, HF, LDA, PBE/GGA) and the experimental value. Different curves were calculated with different basis sets.

A closer look at the calculated data exhibits some trends that help to distinguish bonding contributions estimated by the various levels of theory. The first trend concerns the wellknown underestimation of bond lengths by LDA within the DFT. ${ }^{32}$ An overestimation of bond lengths is found when nonlocal GGA effects are considered. Switching off correlation effects with the HF method also increases the bond lengths. Finally, the predicted bond lengths in $\mathrm{SiP}_{2}$ by B3LYP calculations show a larger deviation from the experiment than LDA, GGA, or even HF. This is rather unexpected, keeping in mind results on molecules and even silicates. ${ }^{33}$ It can be concluded that both non-local effects from GGA and exact exchange from HF result in overestimation of bond lengths. B3LYP sums up both effects that are more expressed for Si-P than for P-P.

As a next step, the bonding situation in $\mathrm{SiP}_{2}$ is analyzed by means of vibrational frequencies (related to bond strength). The pyrite type structure offers a simple identification of stretching modes related to the P-P and Si-P bonds. Calculated vibrational frequencies of selected Raman modes of $\mathrm{SiP}_{2}$ are compared to experimental data in Table II. Respective deviations from the experimental value are visualized in Fig. 3 (only results from the largest basis set are shown). As no experimental IR data is available for $\mathrm{SiP}_{2}$ (IR modes for metallic compounds are highly incapable of measurement), just a comparison with Raman modes is possible at this point. By the correlation of vibrational P-P stretching modes and bond lengths, further clarification of the nature of bonding in the P-P dumbbells should be achieved. The experimental values for the in-phase and out-of-phase P-P stretching modes of 461 and $485 \mathrm{~cm}^{-1}$ (Ref. 28) are in the range of typical values for P-P single bonds. The according stretching mode for

TABLE II. Calculated vibrational frequencies for selected Raman modes.

\begin{tabular}{lccccc}
\hline \hline & $A_{g}$ & $E_{g}$ & $T_{g}^{1}$ & $T_{g}^{2}$ & $T_{g}^{3}$ \\
\hline Expt. $^{28}$ & 461 & 353 & 319 & 337 & 485 \\
LDA & 470 & 364 & 331 & 347 & 494 \\
GGA/PBE & 459 & 357 & 322 & 339 & 484 \\
B3LYP & 480 & 373 & 329 & 355 & 510 \\
HF & 555 & 423 & 387 & 410 & 593 \\
\hline \hline
\end{tabular}

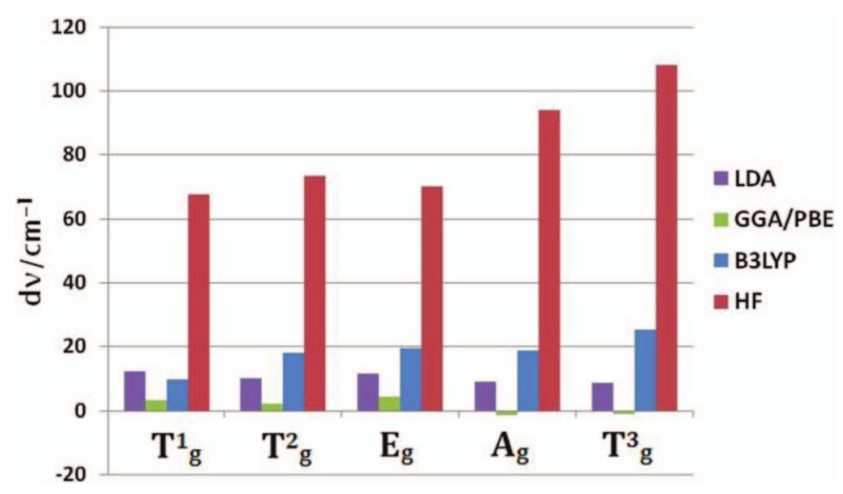

FIG. 3. Deviations of differently calculated Raman modes of $\mathrm{SiP}_{2}$ (LDA, GGA/PBE, B3LYP, and HF) from the experimental value (x-axis).

$\mathrm{P}-\mathrm{P}$ bonds in black phosphorus, for instance, is $470 \mathrm{~cm}^{-1}$. In contrast, the stretching mode of $\mathrm{P}_{2}$ from Raman studies in Ar matrices ${ }^{34}$ is $774 \mathrm{~cm}^{-1}$. Hence, only a slight tendency to higher bond order can be concluded for $\mathrm{SiP}_{2}$.

A general overestimation of the vibrational frequencies can be seen for all levels of theory. Once again, the results from the GGA/PBE method match best with the experimental data. Predicted data from LDA calculations also results in good agreement with the experiment whereas B3LYP performs worse. Hartree-Fock calculations overestimate the frequencies by an order of magnitude compared to LDA. Neglecting correlation for the bond strength and elasticity obviously results in a large error that cannot be seen from bond distances only.

In consideration of these results, the importance of correlation and non-local effects in $\mathrm{SiP}_{2}$ becomes evident. Exact exchange as included in HF or B3LYP does not lead to a better description as it can be observed for molecules or silicates. ${ }^{33}$ The calculated and the experimentally found bond lengths both indicate single bonds within the $\mathrm{P}_{2}$ units. This conclusion supports both ionic $\mathrm{P}_{2}{ }^{4-}$ entities and an entirely covalent network.

The effects of HF and DFT levels of theory should be understood from the electronic structure of $\mathrm{SiP}_{2}$. It is illustrated in "Results II: Electronic structure" section from direct (ELF, AIM) and reciprocal space analyses.

\section{Results II: Electronic structure}

The bonding situation of $\mathrm{SiP}_{2}$ in direct space is shown from the ELF in Fig. 4 for a slide through a phosphorus dumbbell and neighboring $\mathrm{Si}$ atoms. ELF values from 0 to 1 are represented by a color scale from black to red. For all calculations, ELF maxima between two $\mathrm{P}$ atoms of the dumbbell and between $\mathrm{P}$ and $\mathrm{Si}$ atoms indicate covalent bonding. In case of the P-P unit, the ELF maximum is located exactly in the middle of the $\mathrm{P}-\mathrm{P}$ connecting line due to homopolar bonding. Heteropolar bonding with a partially ionic character is anticipated for Si-P. This is underlined by the deviation of the ELF maximum from a connecting line of a $\mathrm{Si}$ and a $\mathrm{P}$ atom. From the point of view of the P-P entity, the ELF maximum tends towards an angle $\left(106^{\circ}\right)$ that is closer to the tetrahedral angle $\left(109^{\circ}\right)$ than the direct line to the next Si atom $\left(104^{\circ}\right)$. A similar 

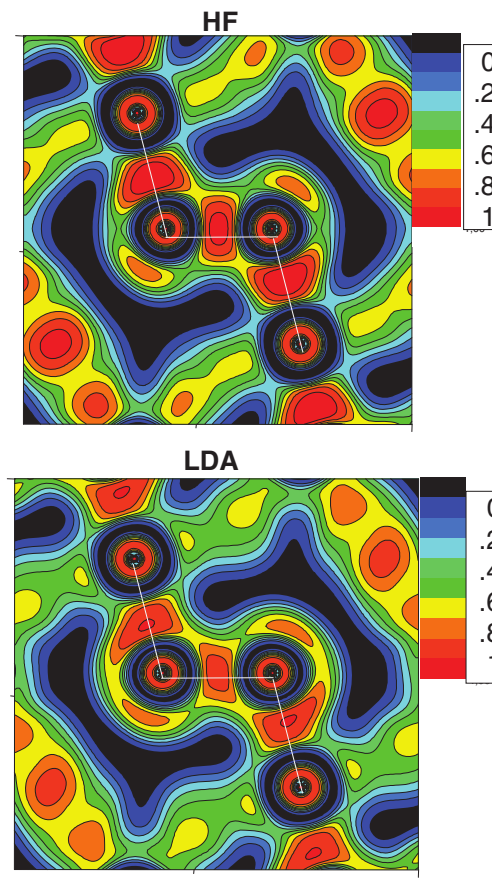
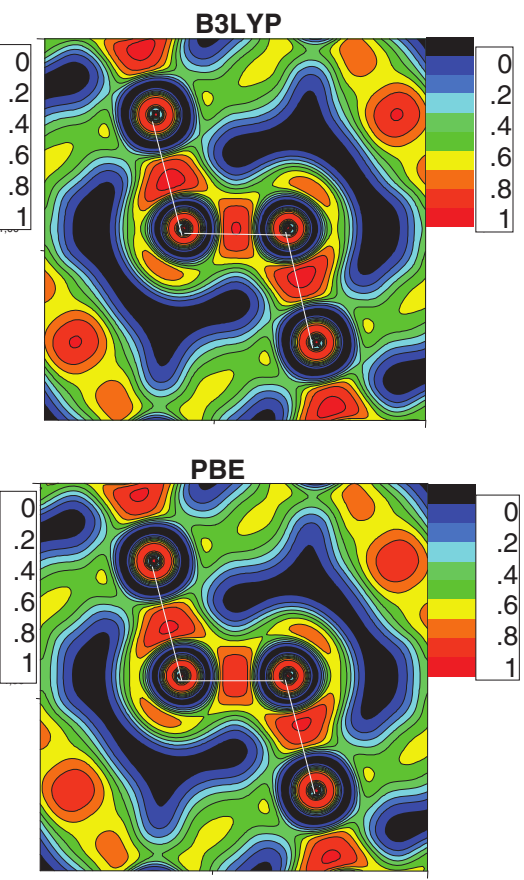

FIG. 4. Electron localization function for $\mathrm{SiP}_{2}$ displayed for a plane containing a $\mathrm{P}_{2}$ dumbbell and two Si atoms (values from 0 to 1 are represented by a color scale from black to red). Calculations based on the HF method are shown as well as calculations based on the DFT functionals B3LYP, LDA, and PBE.

observation was pointed out by von Schnering ${ }^{35}$ from deformation densities based on x-ray and neutron diffraction measurements. He concluded on an ionic character of the bond. This is confirmed by the ELF results that show at least a polarized and bent bond.

Somehow surprisingly, the computed ELF gives very similar results on all levels of theory. The only effect is higher localization for both bonds from HF calculations compared to the results from LDA, GGA, and B3LYP. Thus, the results from the AIM analysis of the charge density according to Bader ${ }^{24,36}$ have to be treated carefully. Beta sphere radii, atomic charges, and volumes of $\mathrm{Si}$ calculated by zero-flux surface integrations as presented in Table III indicate a high charge of +2.5 for $\mathrm{Si}$ according to the HF calculation. In face of a formal valence of +4 , this can be interpreted by assuming a partially covalent character of the Si-P bond. The calculations with the hybrid functional B3LYP as well as LDA and GGA predict lower charges and thus less polarized bonds. The atomic volumes and beta sphere radii follow the same trend. As a hybrid of the mentioned methods, the outcome of the B3LYP functional ranges in between, albeit it is closer to the values obtained by the DFT calculations. Apparently, neglecting electron correlation in favor of an exact exchange energy

TABLE III. Atomic charges, beta sphere radii, and atomic volumes of $\mathrm{Si}$ calculated by zero-flux surface integrations according to Bader's AIM.

\begin{tabular}{lccc}
\hline & $Q(\mathrm{Si}) / \mathrm{e}$ & $V(\mathrm{Si}) / \AA^{3}$ & $r_{B}(\mathrm{Si}) / \AA$ \\
\hline HF & 2.47 & 41.76 & 0.81 \\
B3LYP & 1.50 & 67.04 & 0.93 \\
PBE & 1.39 & 67.96 & 0.94 \\
LDA & 1.39 & 65.09 & 0.91 \\
\hline \hline
\end{tabular}

(HF) leads to a more polarized $\mathrm{Si}-\mathrm{P}$ bond description. However, the differences in the AIM results are simply related to small shifts of the zero flux surface within the Si-P bonding region. Combining ELF and AIM results, one must point out localized bonds for $\mathrm{SiP}_{2}$ from all levels of theory. They are more related to typical covalent bonds found for B-C-Nsystems $^{37}$ than to ionic bonding found in $\mathrm{NaCl}$ where no ELF bonding maxima are found.

Concerning its crystal structure, Raman data, and ELF analysis, $\mathrm{SiP}_{2}$ appears to demonstrate covalent bonding properties (cf. many isostructural pyrite type pnictides that have been classified as semiconductors). Nevertheless, several studies have proven that $\mathrm{SiP}_{2}$ is a compensated conductor. ${ }^{15,38}$ With galvanomagnetic and reflectance measurements, Donohue categorized $\mathrm{SiP}_{2}$ as semimetal with a nearly filled Brillouin zone. A small value of the Hall constant was determined due to the presence of both holes and electrons. Furthermore, conductivity measurements illustrated rising electrical resistivity with increasing temperature. Supplementary, Donohue ${ }^{39}$ performed measurements of the de Haas - van Alphen effect and came up with an energy-level diagram for $\mathrm{SiP}_{2}$. He proposed a completely filled valence band but no bandgap and thus no energy barrier for electrons to transfer to the antibonding band. To come to an analogous conclusion with theoretical investigations, our band structure calculations illustrate how highly the results depend on the calculation method.

So why is $\mathrm{SiP}_{2}$ not a simple semiconductor like $\mathrm{FeS}_{2}$ or even silicates and what was the effect of correlation found from vibrational frequencies? The answer is given by the electronic band structure calculations within HF, B3LYP, PBE/GGA, and LDA levels of theory (Fig. 5). Whereas the constitution and the sequence of the band structures are very 

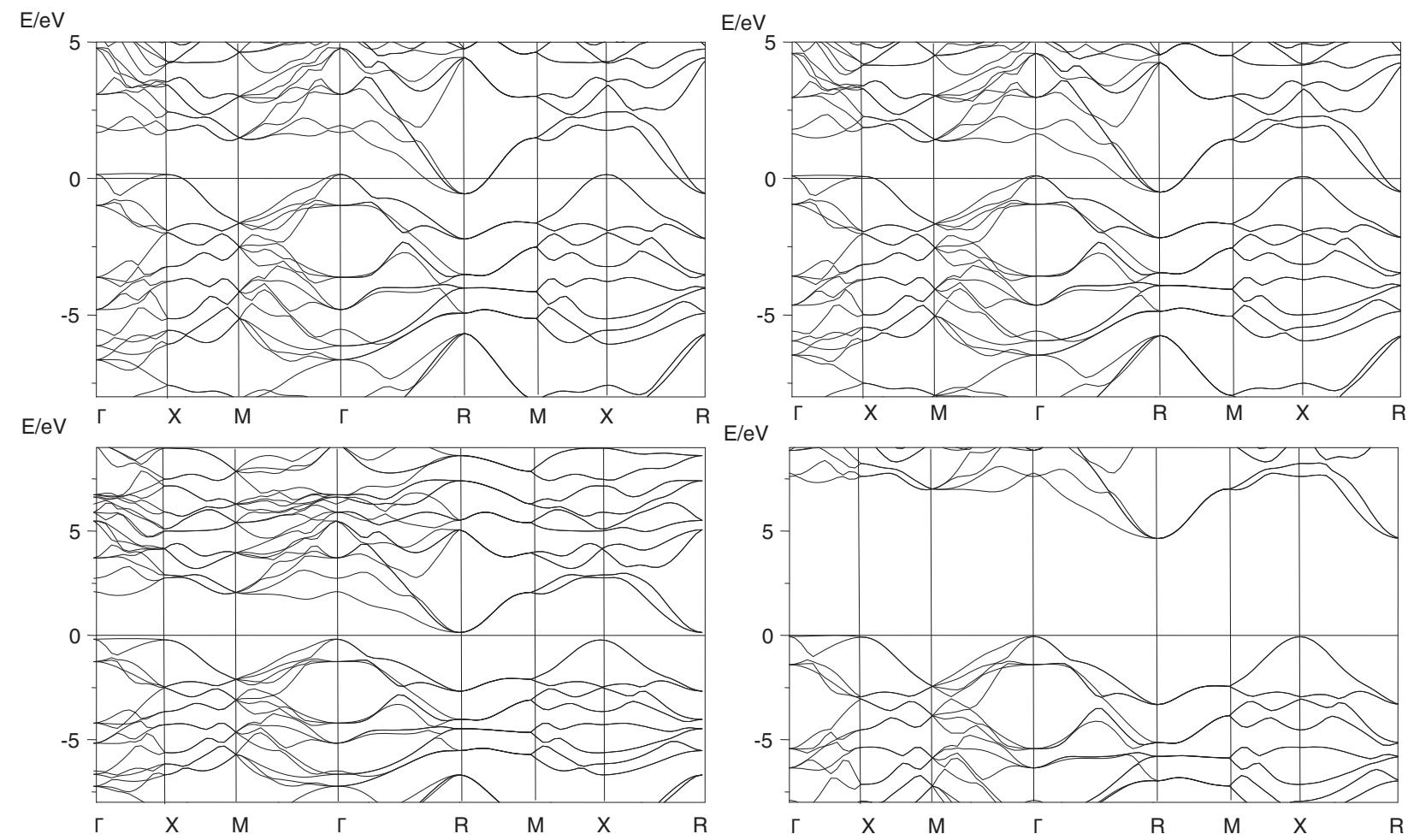

FIG. 5. The band structure of $\mathrm{SiP}_{2}$ - calculated with LDA (top left), GGA (top right), B3LYP (bottom left), and HF (bottom right).

similar, differences are found in the distance between the valence band and the conduction band. LDA and PBE/GGA predict an indirect overlap of $\mathrm{VB}$ and $\mathrm{CB}$ due to a semi metal, B3LYP calculations result in a vanishing and HF in a large bandgap. The gap overestimation by HF is well known but for $\mathrm{SiP}_{2}$ it is crucial as it results in different electronic characteristics. Systems with large gaps between occupied and unoccupied states like molecules or covalent systems like silicates are thus sufficiently described by $\mathrm{HF}$. $\mathrm{SiP}_{2}$, on the other hand, is found on the edge between covalent and delocalized metallic bonding.

Keeping this in mind, the good agreement of LDA and GGA predictions with experimental data is due to the fact that they consider both covalent and metallic characteristics. In line with the results in Ref. 40, the properties of $\mathrm{SiP}_{2}$ are dominated by its semimetallic properties. But why is $\mathrm{SiP}_{2}$ a semimetal with an indirect overlap of valence and conduction bands? Why does it not form a gap like $\mathrm{FeS}_{2}$ or as predicted from HF? As an answer, the present calculations show a flat band segment between the $\Gamma$ and the $\mathrm{X}$ point that has not been reported previously. It indicates instability due to antibonding or nonbonding states. Related band segments at the Fermi level were also related to the appearance of superconductivity. ${ }^{41}$ It seems that the occupation of this valence band region is avoided in $\mathrm{SiP}_{2}$ and it is energetically better to occupy the minimum of the conduction band at the $\mathrm{R}$ point.

An analysis of the band characteristics was carried out with the aid of projected DOS calculations (PDOS) based on LDA (Fig. 6). The $3 s, 3 p$, and $3 d$ orbitals of silicon and phosphorus are displayed in addition to the total DOS. In accor- dance with OPW DOS calculations and experimental x-ray emission bands of Farberovich, ${ }^{40}$ the total DOS generates five maxima below the Fermi level.

The lowest three maxima for silicon result from $3 s$, the one at about $-4 \mathrm{eV}$ from $3 p$, and the one close to the Fermi energy from $3 d$ contributions. These $3 d$ contributions are required to explain the distorted octahedral coordination of silicon atoms in $\mathrm{SiP}_{2}$ as an $s p^{3} d^{2}$ hybridization for $\mathrm{Si}$ has to be assumed in this case. An $s p^{3} d^{2}$ hybridization for $\mathrm{Si}$ was already predicted by Donohue in 1969 (Ref. 39) to explain the unusual 6-fold coordination of Si.

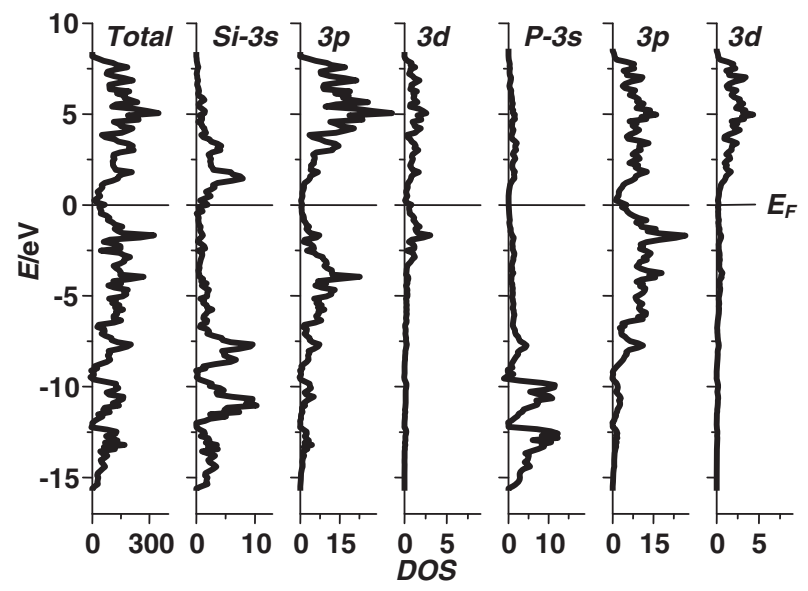

FIG. 6. Density of states (DOS) and projected density of states (PDOS) of $\mathrm{Si}$ and $\mathrm{P}$ in $\mathrm{SiP}_{2}$. 


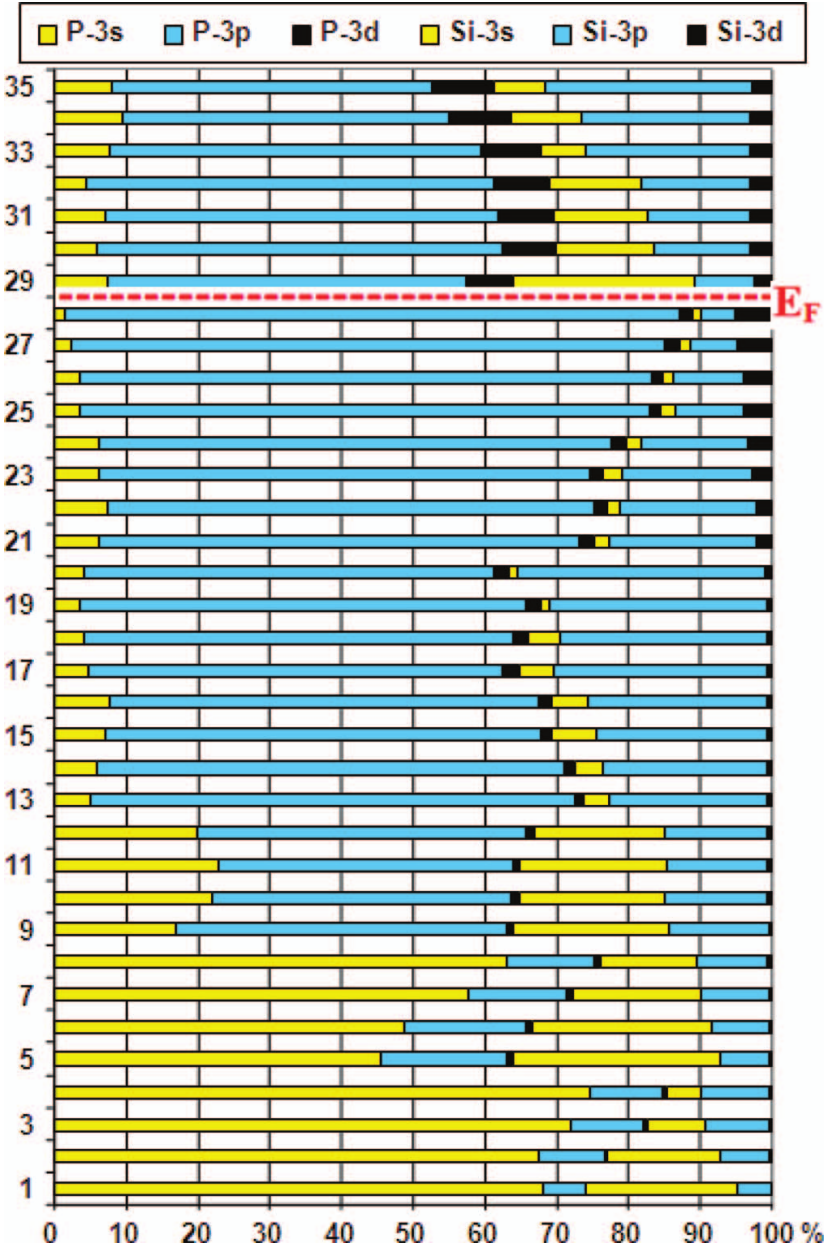

FIG. 7. Projection of the $\mathrm{P}$ and $\mathrm{Si} s, p$, and $d$ fractions of the respective bands in $\mathrm{SiP}_{2}$ obtained from a numerical integration of the projected density of states of the individual bands.

The two strong maxima between -15 and $-10 \mathrm{eV}$ of the phosphorus $3 s$ states confer to $\sigma \mathrm{P}-\mathrm{P}$ and $\sigma$ Si-P bonds. P- $3 p$ states are also located below the Fermi level and participate in $\sigma$ and $\pi$ bonds to neighboring atoms. The $3 d$ states of the phosphorus PDOS plot are above the Fermi level so they do not participate in any binding states. This is coherent with the predicted distorted tetrahedral coordination of $\mathrm{P}$ atoms in $\mathrm{SiP}_{2}$ and it indicates an $s p^{3}$ hybridization of $\mathrm{P}$ atoms.

To gain deeper insight into band contributions of the atomic orbitals, a detailed breakdown according to the PDOS of the individual bands is presented in Fig. 7. The present approach separates distinct bands along the E scale. Thus, band overlap is not disturbing like in the PDOS picture. Bands one to 35 (of 56) are taken into consideration; they are subdivided into six sections which point out the respective percentages of the $3 s, 3 p$, and $3 d$ orbitals of phosphorus and silicon. The edges of the VB and the $\mathrm{CB}$ are given between bands 28 and 29 . This picture refers to the semiconducting limit predicted from B3LYP where 28 bands are occupied by 56 valence electrons per formula unit (Si: 4VE, P: 5VE, $4 \mathrm{FU}$ ). In agreement with the PDOS (Fig. 6), mostly $s$ character is indicated in the first block of eight bands due to $\sigma$ and $\sigma *$ bonds of the P-P dumbbells. The overlaying bands predominantly demon- strate $p$ character according to significant $p$ contributions in the bonds of $s p^{3}$ and $s p^{2} d^{3}$ hybridized $\mathrm{P}$ and $\mathrm{Si}$ atoms. Below the Fermi level, there is only little overall $d$ character; especially phosphorus barely donates any binding $d$ contributions. Nevertheless, an increasing $\mathrm{Si} d$ character below the Fermi level is a sign of the distorted octahedral coordination of silicon atoms, which requires $s p^{2} d^{3}$ hybridization. Passing the Fermi level, an abrupt rise of the Si $s$ character refers to distinctive antibonding states. One must conclude that the metallic characteristics are due to the occupation of bands that are related to $\mathrm{Si}-3 s$ and $\mathrm{P}-3 p$ states. This is preferred over the occupation of nearly pure $\mathrm{P}-3 p$ states in the flat valence band region. A reason might be $\mathrm{P}-\mathrm{P}$-interaction as estimated by von Schnering, ${ }^{29,35} \mathrm{cf}$. the conduction band minimum that could be caused by bonding of $\mathrm{Si}-3 s$.

\section{CONCLUSION}

The present work is dedicated to a detailed investigation of the ambivalent bonding situation in pyrite type $\mathrm{SiP}_{2}$. First principles calculations were performed on various levels of theory including DFT-LDA/GGA, HF, and hybrid B3LYP to understand the different bonding contributions. From all calculations, bond distances were predicted in very good agreement with experimental data. The same is found for Raman frequencies with the exception of HF. Coordination, bond distances, and vibrational frequencies are in line with covalent or ionic models according to a Zintl description $\mathrm{Si}\left[\mathrm{P}_{2}\right]$. Real space analysis by the ELF indicates typical covalent P-P and polarized P-Si bonds. The reason for the different bond descriptions by the different methods of calculation becomes apparent when the band structures are taken into consideration. HF and B3LYP predict $\mathrm{SiP}_{2}$ to be a semiconductor analogously to $\mathrm{FeS}_{2}$. Only LDA and GGA predict a semimetallike indirect overlap of the valence and the conduction band in agreement with experimental data. This behavior can be explained by a flat band region in the valence band of $\mathrm{SiP}_{2}$. It is caused by antibonding P-3p states on top of the VB while the conduction band minimum is lowered by $\mathrm{Si}-3 s-\mathrm{P}-3 p$ interactions. Further investigations concerning $\mathrm{SiP}_{2}$ could focus on the flat band segment between $\Gamma$ and $X$. If it can be lowered to the Fermi level, interesting properties like super-conductivity or spin-polarization could be observed.

\section{ACKNOWLEDGMENTS}

The authors would like to thank Professor A. Pfitzner and the Deutsche Forschungsgemeinschaft (DFG, SPP1415) for financial support.

This paper is dedicated to Carla Roetti, deceased September 7, 2010.

${ }^{1}$ M. Rück, D. Hoppe, B. Wahl, P. Simon, Y. K. Wang, and G. Seifert, Angew. Chem., Int. Ed. 44(46), 7616 (2005).

${ }^{2}$ A. Pfitzner, Angew. Chem., Int. Ed. 45(5), 699 (2006).

${ }^{3}$ A. Pfitzner, M. F. Brau, J. Zweck, G. Brunklaus, and H. Eckert, Angew. Chem., Int. Ed. 43(32), 4228 (2004).

${ }^{4}$ S. Lange, M. Bawohl, R. Weihrich, and T. Nilges, Angew. Chem., Int. Ed. 47(30), 5654 (2008).

${ }^{5}$ E. Freudenthaler and A. Pfitzner, Z. Kristallogr. 212(2), 103 (1997). 
${ }^{6}$ E. Freudenthaler and A. Pfitzner, Solid State Ionics 101-103(Pt. 2), 1053 (1997).

${ }^{7}$ T. Wadsten, Acta Chem. Scand. 21(2), 593 (1967); 21(5), 1374 (1967).

${ }^{8}$ R. Weihrich, V. Eyert, and S. F. Matar, Chem. Phys. Lett. 373(5,6), 636 (2003).

${ }^{9}$ J. Osugi, R. Namikawa, and Y. Tanaka, Rev. Phys. Chem. Jpn. 36(1), 35 (1966).

${ }^{10}$ P. C. Donohue, W. J. Siemons, and J. L. Gillson, J. Phys. Chem. Solids 29(5), 807 (1968).

${ }^{11}$ E. Zintl and G. Brauer, Z. Phys. Chem. 20B, 245 (1933).

${ }^{12}$ T. Chattopadhyay, A. Werner, and H. G. von Schnering, J. Phys. Chem. Solids 44(7), 699 (1983).

${ }^{13}$ O. V. Farberovich, Sov. Phys. Semicond. 13(10), 1171 (1979).

${ }^{14}$ E. P. Domashevskaya, L. N. Marshakova, V. A. Terekhov, Y. A. Ugai, and O. V. Farberovich, Tezisy Dokl.-Vses. Konf. Khim. Svyazi Poluprovodn. Polumetallakh 5, 53 (1974)

${ }^{15}$ E. P. Domashevskaya, L. N. Marshakova, V. A. Terekhov, Y. A. Ugai, and O. V. Farberovich, Khim. Svyaz' v Kristallakh i ikh Fiz. Svoistva. 1, 84 (1976).

${ }^{16}$ M. Meier and R. Weihrich, Chem. Phys. Lett. 461(1-3), 38 (2008).

${ }^{17}$ R. Dovesi, crystal98 User's Manual (University of Torino, Torino, 1998).

${ }^{18} \mathrm{R}$. Dovesi, crystal09 User's Manual (University of Torino, Torino, 2006).

${ }^{19}$ L. W. S. H. Vosko and M. Nusair, Can. J. Phys. 58, 1200 (1980).

${ }^{20}$ J. P. Perdew, K. Burke, and M. Ernzerhof, Phys. Rev. Lett. 77(18), 3865 (1996).

${ }^{21}$ A. D. Becke, Phys. Rev. A 38(6), 3098 (1988); C. Lee, W. Yang, and R. G. Parr, Phys. Rev. B 37(2), 785 (1988).

${ }^{22}$ P. Hohenberg and W. Kohn, Phys. Rev. 136(3B), B864 (1964).

${ }^{23}$ D. R. Hartree, Proc. Cambridge Philos. Soc. 24, 89 (1928).

${ }^{24}$ R. F. W. Bader, Atoms in Molecules - A Quantum Theory (Oxford University Press, New York, 1990).
${ }^{25}$ C. Gatti, TOPOND96 User's Manual (CNR-CSRSRC, Milan, 1997).

${ }^{26}$ D. G. Anderson, J. ACM 12(4), 547 (1965).

${ }^{27}$ A. C. Stückl, R. Weihrich, and K. J. Range, Frontiers of Solid State Chemistry, Proceedings of the International Symposium on Solid State Chemistry in China, Changchun, China, August 9-12, 2002, pp. 117-124.

${ }^{28}$ H. Vogt, T. Chattopadhyay, and H. J. Stolz, J. Phys. Chem. Solids 44(9), 869 (1983).

${ }^{29}$ T. K. Chattopadhyay and H. G. von Schnering, Z. Kristallogra. 167(1-2), 1 (1984).

${ }^{30}$ H. Okudera, R. E. Dinnebier, and A. Simon, Z. Kristallogr. 220(2-3), 259 (2005).

${ }^{31}$ L. Cartz, S. R. Srinivasa, R. J. Riedner, J. D. Jorgensen, and T. G. Worlton, J. Chem. Phys. 71(4), 1718 (1979).

${ }^{32}$ K. Seifert, J. Hafner, J. Furthmuller, and G. Kresse, J. Phys. Condens. Matter 7(19), 3683 (1995).

${ }^{33}$ C. M. Zicovich-Wilson, F. Pascale, C. Roetti, V. R. Saunders, R. Orlando, and R. Dovesi, J. Comput. Chem. 25(15), 1873 (2004).

${ }^{34}$ A. Kornath, A. Kaufmann, and M. Torheyden, J. Chem. Phys. 116(8), 3323 (2002).

${ }^{35}$ T. Chattopadhyay and H. G. von Schnering, Stud. Inorg. Chem. 3, 761 (1983).

${ }^{36}$ R. F. W. Bader and P. M. Beddall, J. Am. Chem. Soc. 95(2), 305 (1973); R. F. W. Bader, J. Hernandez-Trujillo, and F. Cortes-Guzman, J. Comput. Chem. 28(1), 4 (2007).

${ }^{37}$ R. Weihrich, S. F. Matar, E. Betranhandy, and V. Eyert, Solid State Sci. 5(5), 701 (2003).

${ }^{38}$ O. V. Farberovich and E. P. Domashevskaya, Sov. Phys. Semicond. 10(6), 1193 (1976).

${ }^{39}$ S. M. Marcus and P. C. Donohue, Phys. Rev. 183(3), 668 (1969).

${ }^{40}$ O. V. Farberovich and E. P. Domashevskaya, Sov. Phys. Semicond. 9(5), 1001 (1975).

${ }^{41}$ A. Simon, Angew. Chem., Int. Ed. 36(17), 1789 (1997). 